\title{
Self-rated sleep quality and mood states of Brazilian fighters
}

\author{
Guilherme Guimarães BEVILACQUA*, Rodrigo Batalha SILVA, Verônica Maria CLAUDINO, \\ Bianca dos Santos SEVERINO, Maria Eduarda Soares RODRIGUES, \& Alexandro ANDRADE \\ Laboratory of Sports and Exercise Psychology - Santa Catarina State University (Brazil)
}

8th IMACSSS International Conference Abstracts, Viseu (Portugal), October 10-12, 2019

Type: Poster presentation

\begin{abstract}
Sleep has a direct relationship with mood. In this context, the aim of this study was to analyze the relationship between sleep quality and mood of combat sport athletes. A total of 188 elite male combat sports athletes participated in the study. Athletes who reported good sleep have greater vigor during competition than athletes with regular sleep and the mood profile was similar to the Iceberg Profile. In conclusion, there are relationships between sleep quality and mood of combat sport athletes, and athletes with good sleep quality present a mood profile nearest to the ideal for the best performance.
\end{abstract}

Keywords: Sport psychology; combat sports; martial arts; sleep; mood.

\section{Introduction}

Sports performance is the result of a series of variables, including sleep, which is responsible for bodily rest and recovery of physical and cognitive abilities (Davenne, 2009). Just like sleep, psychological variables are important for the sports performance of elite athletes. Among these psychological factors, mood has been highlighted in recent research (Brandt, Bevilacqua, \& Andrade, 2017; Chennaoui et al., 2016). Mood is an emotional construct that can vary in intensity and duration and is composed of six different states: tension, anger, depression, fatigue, confusion and vigor (Lane \& Terry, 2000). Studies have shown that overall performance is associated with low levels of tension, anger, depression, fatigue and confusion and high vigor (Andrade, Bevilacqua, Coimbra, Pereira, \& Brandt, 2016). Sleep has a direct relationship with mood (Lastella, Lovell, \& Sargent, 2014) and sports performance. In this context, in combat modalities, different aspects about the relations between mood, sleep and sports performance are not consolidated in the literature, presenting itself as an important research gap.

\section{Objectives}

The aim of this study was to analyze the relationship between sleep quality and mood of combat sport athletes.

\section{Methodology}

A total of 188 elite male combat sports athletes participated in the study ( $M_{\text {age }}=26.20 \pm$ 7,29 years). Athletes from four combat sports were surveyed, being 118 Brazilian Jiu-Jitsu (62,8\% / $M_{\text {age }}=28.26 \pm 7.32$ years $), 47$ judo athletes $\left(25 \% / M_{\text {age }}=23.04 \pm 5.15\right.$ years $), 15$ taekwondo athletes $\left(8 \% / M_{\mathrm{age}}=22.50 \pm 4.67\right.$ years $)$ and 8 karate athletes $\left(4.2 \% / M_{\mathrm{age}}=21.86 \pm 11.53\right.$ years $)$.

The athletes were classified as elite athletes following the criteria of Swann, Moran and Piggott (2015), with 28\% ( $n=44)$ competing at the state level, 29.9\% $(n=47)$ at the national level and $42 \%(n=66)$ at the international level. A questionnaire was used for general characterization of the athletes and the Brunel Mood Scale - BRUMS was used to verify the athletes' mood states. Sleep was assessed through a self-reported question "How do you self-assess the quality of your

\footnotetext{
${ }^{*}$ Email: alexandro.andrade.phd@gmail.com
} 
sleep?". The answer options were based on a Likert scale (from $0=$ poor to $4=$ excellent). Data was treated using descriptive and inferential statistics trough SPSS software version 20.0. The data were analyzed using descriptive statistics (mean, standard deviation, frequency, and percentage) and inferential statistics. Inferential analysis was performed using the Kruskal-Wallis test to compare the mood of athletes with different self-reported sleep quality. When differences were found, we used Dunn's post hoc test. This study was approved by the Committee for Ethics in Human Research of the Santa Catarina State University under protocol 44/2011.

\section{Results}

The Kruskal-Wallis test showed that there was a difference in vigor between athletes with different levels of sleep quality. $\left(\chi^{2}(4)=17.010, p=0,002 ; \eta^{2}=0.079\right.$; Effect Size [ES]: 0.574, small). After Dunn's post hoc, it was observed that athletes who reported good sleep had greater vigor during competition than athletes with regular sleep. There was no significant difference in the other comparisons. However, it can be observed that athletes who reported poor sleep have a worse Iceberg Profile, with higher tension, depression, anger, fatigue and confusion and lower vigor (Figure 1).

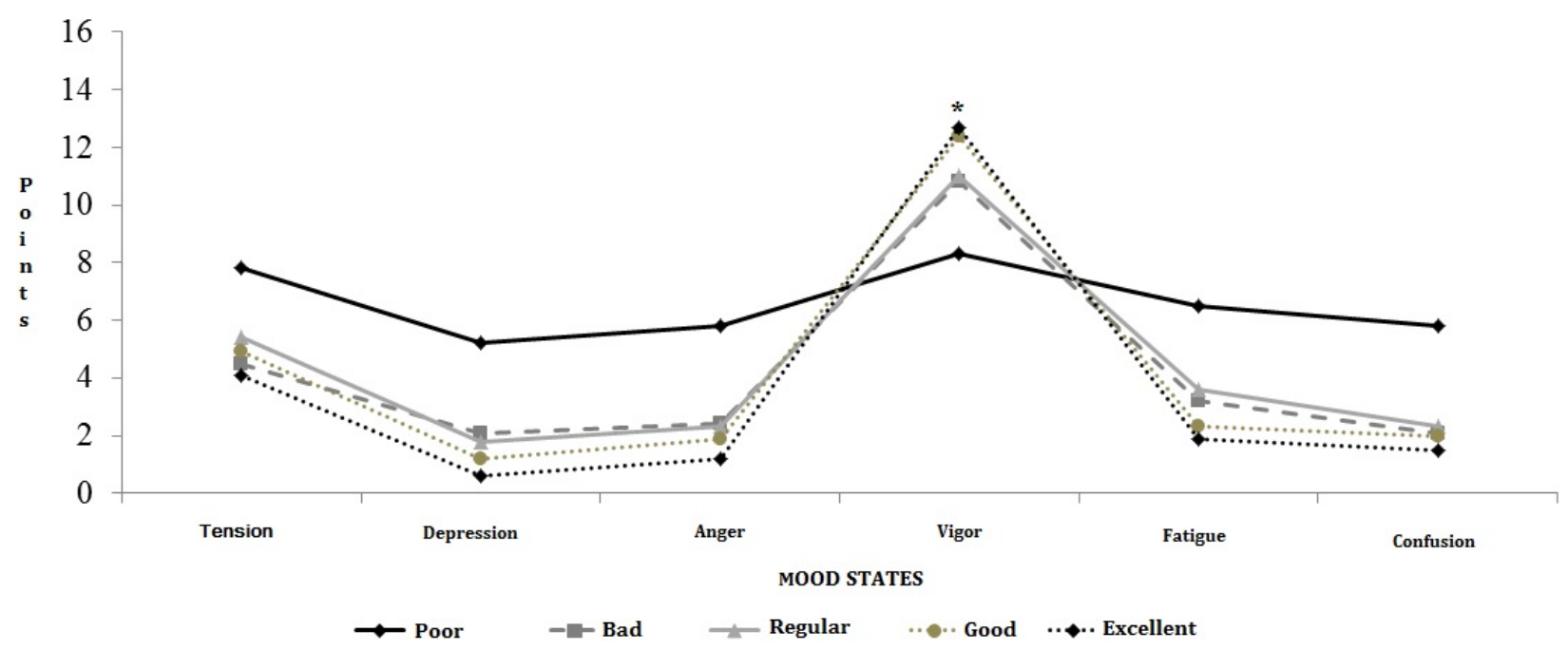

Figure 1. Mood of male combat sports athletes with different levels of sleep quality. *Difference between groups $(p<0.002)$ by Kruskal-Wallis test, post hoc Dunn. Values on average.

\section{Discussion}

In our study we found that athletes who reported good sleep had greater vigor during competition than athletes who had regular sleep. These results are similar to the study by Andrade, Bevilacqua, Casagrande, Brandt and Coimbra (2018), in which athletes who rated excellent sleep had greater vigor compared to athletes with regular or poor sleep. In general, combat sports athletes exhibited mood similar to Iceberg Profile. Although without significant differences, we observed a tendency for athletes with poor sleep to have significant changes in mood profile, with levels of negative mood factors close to vigor. Studies indicate that a positive Iceberg Profile may be an indicator for better sports performance (Lane \& Terry, 2000), with high vigor and low levels of tension, depression, anger, confusion and fatigue. However, the "ideal" mood profile may vary by sport. For combat sports, studies show that elevated anger coupled with vigor can have a facilitating effect on increased activation levels (Brandt et al., 2015). However, studies investigating these modalities are scarce. In the case of Brazilian jiu-jitsu, anger at moderate levels can be considered positive as it can postpone fatigue by altering bodily perceptions (Tenenbaum \& Eklund, 2007).

\section{Conclusion}

It can be concluded that there are relationships between sleep quality and mood of combat sport athletes, and athletes with good sleep quality present a mood profile closer to the ideal for the best performance. 


\section{References}

Andrade, A.; Bevilacqua, G. G.; Casagrande, P. O.; Brandt, R.; Coimbra, D. R. (2018). Sleep quality associated with mood in elite athletes. The Physician and Sportsmedicine, in press. doi: $\underline{10.1080 / 00913847.2018 .1553467}$

Andrade, A., Bevilacqua, G. G., Coimbra, D. R., Pereira, F. S., \& Brandt, R. (2016). Sleep quality, mood and performance: A study of elite Brazilian volleyball athletes. Journal of Sports Science and Medicine, 15(4), 601-605.

Brandt, R., Bevilacqua, G. G., \& Andrade, A. (2017). Perceived Sleep Quality, Mood States, and Their Relationship with Performance Among Brazilian Elite Athletes During a Competitive Period. Journal of Strength and Conditioning Research, 31(4), 1033-1039. doi: 10.1519/ISC.0000000000001551

Brandt, R., Hobold, E., Viana, M. da S., Dominski, F. H., Bevilacqua, G. G., \& Turczyn, B. (2015). Humor pré-competitivo em atletas brasileiros de jiu-jitsu. Caderno de Educação Física e Esporte, 13(1), 21-30.

Chennaoui, M., Bougard, C., Drogou, C., Langrume, C., Miller, C., Gomez-Merino, D., \& Vergnoux, F. (2016). Stress Biomarkers, Mood States, and Sleep during a Major Competition: "Success" and "Failure" Athlete's Profile of High-Level Swimmers. Frontiers in Physiology, 7, 94. doi: 10.3389/fphys.2016.00094

Davenne, D. (2009). Sleep of athletes - Problems and possible solutions. Biological Rhythm Research, 40(1), 45-52. doi: 10.1080/09291010802067023

Lane, A. M., \& Terry, P. C. (2000). The Nature of Mood: Development of a Conceptual Model with a Focus on Depression. Journal of Applied Sport Psychology, 12(1), 16-33. doi: $10.1080 / 10413200008404211$

Lastella, M., Lovell, G. P., \& Sargent, C. (2014). Athletes' precompetitive sleep behaviour and its relationship with subsequent precompetitive mood and performance. European Journal of Sport Science, 14(sup1), S123-S130. doi: 10.1080/17461391.2012.660505

Swann, C., Moran, A., \& Piggott, D. (2015). Defining elite athletes: Issues in the study of expert performance in sport psychology. Psychology of Sport and Exercise, 16(1), 3-14. doi: 10.1016/J.PSYCHSPORT.2014.07.004

Tenenbaum, G., \& Eklund, R. C. (2007). Handbook of Sport Psychology (3rd Ed.). Hoboken, New Jersey: John Wiley \& Sons. doi: $10.1002 / 9781118270011$ 